\title{
On The Sublattice [I, C0] Of the Lattice of Cech Closure Operators
}

\author{
Baby Chacko \\ Associate Professor, Department of Mathematics, St.Joseph's College, Devagiri, Calicut-8, and Kerala, India.
}

\begin{abstract}
The interval $\left[I, C_{0}\right]$ where $I$ is the indiscrete closure operator and $C_{0}$ is the co-finite closure operator on a set $X$ is a complete sublattice of the lattice of all closure operators on $X$. In this paper, we determine a class of automorphisms of the lattice $\left[I, C_{0}\right]$ and characterize the group of automorphisms on $\left[I, C_{0}\right]$ when $X$ is finite.
\end{abstract}

Mathematics Subject Classification: 54A05

Keywords: lattice of all closure operators on a set X, infra closure operator, atomistic lattice, lattice isomorphism, lattice automorphism, reflexive relation.

\section{Introduction}

In 1958, Juris Hartmanis [4] determined the automorphisms of the lattice LT(X) of all topologies on a fixed set $X$ as follows : for $p \in S(X)$ and $\tau \in L T(X)$, define the mapping $A_{p}$ by $A_{p}(\tau)=\{p(U): U \in \tau\}$. Then $A_{p}(\tau)$ is a topology on $X$ and $A_{p}(\tau)$ is an automorphism of $L T(X)$. If $X$ is infinite or $X$ contains atmost two elements, the group of automorphisms of $L T(X)$ is precisely $\left\{A_{p}: p \in S(X)\right\}$. Otherwise, the group of automorphisms of $\operatorname{LT}(X)$ is $\left\{\mathrm{A}_{\mathrm{p}}: \mathrm{p} \in \mathrm{S}(\mathrm{X})\right\} \cup\left\{\mathrm{B}_{\mathrm{p}}: \mathrm{p} \in \mathrm{S}(\mathrm{X})\right\}$ where $\mathrm{B}_{\mathrm{p}}: \operatorname{LT}(X) \rightarrow \operatorname{LT}(X)$ is defined by $\mathrm{B}_{\mathrm{p}}(\tau)$ $=\{X-p(U): U \in \tau\}$ for $\tau \in L T(X)$. From this result, we can conclude that, if $X$ is an infinite set and $P$ is any topological property, then the set of topologies in $\mathrm{LT}(\mathrm{X})$ possessing the property $\mathrm{P}$ may be identified simply from the lattice structure of $\mathrm{LT}(\mathrm{X})$, since the only automorphisms of LT(X) for infinite $X$ are those which simply permute elements of X. Therefore any automorphism of LT(X) must map all the topologies in LT(X) onto their homeomorphic images. Thus the topological properties of elements of $\mathrm{LT}(\mathrm{X})$ must be determined by the position of the topologies in $\mathrm{LT}(\mathrm{X})$. In this paper, we determine a class of automorphisms of the lattice [I, $\left.\mathrm{C}_{0}\right]$ and characterize the group of automorphisms on $\left[\mathrm{I}, \mathrm{C}_{0}\right]$ when $\mathrm{X}$ is finite.

\section{Definition 2.1}

\section{Preliminaries}

Let $\wp(X)$ denotes the power set of a set $\mathrm{X}$. A Cech closure operator on a set $\mathrm{X}$ is a function $\mathrm{V}: \wp(\mathrm{X})$ $\rightarrow \wp(\mathrm{X})$ such that,

(i) $\mathrm{V}(\phi)=\phi$

(ii) $\mathrm{A} \subseteq \mathrm{V}(\mathrm{A})$ for all $\mathrm{A} \in \wp(\mathrm{X})$

(iii) $\mathrm{V}(\mathrm{A} \cup \mathrm{B})=\mathrm{V}(\mathrm{A}) \cup \mathrm{V}(\mathrm{B})$ for all $\mathrm{A}, \mathrm{B} \in \wp(\mathrm{X})$.

For brevity, we call $\mathrm{V}$ a closure operator on $\mathrm{X}$ and the pair $(\mathrm{X}, \mathrm{V})$ a closure space.

Definition 2.2

Let $(\mathrm{X}, \mathrm{V})$ be a closure space. A subset $\mathrm{A}$ of $\mathrm{X}$ is said to be closed, if $\mathrm{V}(\mathrm{A})=\mathrm{A}$ and open, if $\mathrm{V}(\mathrm{X}-\mathrm{A})=\mathrm{X}$ A.

\section{Definition 2.3}

The set of all open sets in $(\mathrm{X}, \mathrm{V})$ is a topology on $\mathrm{X}$, called the topology associated with $\mathrm{V}$. On the other hand, to every topology $\tau$ on $\mathrm{X}$, we can associate a closure operator $\mathrm{V}$ on $\mathrm{X}$ (the Kuratowski closure operator) defined by $\mathrm{V}(\mathrm{A})=\operatorname{cl}(\mathrm{A})$ where $\operatorname{cl}(\mathrm{A})$ denotes the closure of $\mathrm{A}$ in $(\mathrm{X}, \tau)$. We say that $\mathrm{V}$ is the closure operator associated with $\tau$.

\section{Remark 2.4}

A closure operator on a set need not be the closure operator associated with the topology associated with it. In this sense Cech closure operators on a set X can be considered as generalization of topologies on X.

\section{Definition 2.5}

Let $V_{1}$ and $V_{2}$ be two closure operators on a set $X$. Then $V_{1}$ is said to " coarser than " $V_{2}$ ( or $V_{2}$ is said to finer than $\left.V_{1}\right)$ if $V_{1}(A) \supseteq V_{2}$ for all $A \in \wp(X)$. In this case we write $V_{1} \leq V_{2}$.

\section{Example 2.6}

Let $\quad \mathrm{V}: \wp(\mathrm{X}) \rightarrow \wp(\mathrm{X})$ be defined by

$$
\begin{aligned}
\mathrm{V}(\mathrm{A}) & =\phi \text { if } \mathrm{A}=\phi \\
& =\mathrm{X} \text { otherwise. }
\end{aligned}
$$


Then $\mathrm{V}$ is a closure operator on $\mathrm{X}$, called the indiscrete closure operator. The indiscrete closure operator is usually denoted by $\mathrm{I}$.

\section{Example 2.7}

Let $\mathrm{X}$ be an infinite set. Define $\mathrm{V}: \wp(\mathrm{X}) \rightarrow \wp(\mathrm{X})$ by

$$
\begin{aligned}
\mathrm{V}(\mathrm{A}) & =\mathrm{A} \text { if } \mathrm{A} \text { is finite, } \\
& =\mathrm{X} \text { otherwise. }
\end{aligned}
$$

Then $\mathrm{V}$ is a closure operator on $\mathrm{X}$, called the co-finite closure operator. The co-finite closure operator is usually denoted by $\mathrm{C}_{0}$.

\section{Remark 2.8}

The relation " coarser than " is a partial order on the set of all closure operators on X. We denote the set of all closure operators on a set $\mathrm{X}$ by $\mathrm{LC}(\mathrm{X})$. Then $\mathrm{LC}(\mathrm{X})$ is a complete lattice under the relation "coarser than " and the least element of this lattice is $\mathrm{I}$.

\section{Definition 2.9}

A closure operator on $\mathrm{X}$ other than I is called an infra closure operator, if the only closure operator on $\mathrm{X}$ strictly smaller than it is I, the indiscrete closure operator on X. Note that the infra closure operators on X are precisely the atoms of the lattice $\mathrm{LC}(\mathrm{X})$.

\section{Definition 2.10}

$$
\begin{aligned}
& \text { For } a, b \in X, a \neq b \text {, define } V_{(a, b)} \text { by, } \\
& \begin{aligned}
V_{(a, b)}(A) & =A \text { if } A=\phi, \\
& =X-\{b\} \text { if } A=\{a\}, \\
& =X \text { otherwise. }
\end{aligned}
\end{aligned}
$$

Then $\mathrm{V}_{(\mathrm{a}, \mathrm{b})}$ is an infra closure operator on $\mathrm{X}$.

\section{Theorem 2.11}

A closure operator on $\mathrm{X}$ is an infra closure operator if and only if it is of the form $\mathrm{V}_{(\mathrm{a}, \mathrm{b})}$ for some $\mathrm{a}, \mathrm{b} \in \mathrm{X}$ such that $a \neq b$. [6]

Notation 2.12

We use the notation $\Omega$ to denote the atoms of the lattice $\operatorname{LC}(\mathrm{X})$. Then by the Theorem 1 , the members of $\Omega$ are of the form $\mathrm{V}_{(\mathrm{a}, \mathrm{b})}$ where $\mathrm{a}, \mathrm{b} \in \mathrm{X}, \mathrm{a} \neq \mathrm{b}$.

\section{Remark 2.13}

The interval $\left[I, C_{0}\right]$ where $I$ is the indiscrete closure operator and $\mathrm{C}_{0}$ is the co-finite closure operator on a set $\mathrm{X}$ is a complete sublattice of the lattice $\mathrm{LC}(\mathrm{X})$.

\section{Remark 2.14}

The set of all closure operators [I, $C_{0}$ ] on a set $X$ under the partial order " $\leq$ " defined by $V_{1} \leq V_{2} \Leftrightarrow V_{2}$ (A) $\subseteq \mathrm{V}_{1}(\mathrm{~A})$ for every $\mathrm{A} \in \wp(\mathrm{X})$ is a complete lattice.

\section{Notation 2.15}

We use the notation $\mathrm{S}(\mathrm{X})$ to denote the set of all bijections on $\mathrm{X}$.

\section{Lemma 3.1}

\section{Main Results}

For a closure operator $\mathrm{V}$ on $\mathrm{X}$ such that $\mathrm{V} \leq \mathrm{C}_{0}$, define a relation $\rho \mathrm{V}$ on $\mathrm{X}$ by $\rho \mathrm{V}=\{(\mathrm{x}, \mathrm{y}): \mathrm{y} \in \mathrm{V}(\{\mathrm{x}\})\}$. Then $\rho \mathrm{V}$ is a reflexive relation on $\mathrm{X}$.

Proof : Obvious.

Lemma 3.2

For $R \in L R(X)$, the lattice of all reflexive relation on $X$, define $v R: \wp(X) \rightarrow \wp(X)$ by $v R(A)=\{y \in X$ : $x R y$ for some $x \in A\}, A \in \wp(X)$. Then $u R$ is a closure operator in $\left[I, C_{0}\right]$.

Proof: Obvious.

Remark 3.3

It can be easily verified that, the mapping $v: L R(X) \rightarrow\left[I, C_{0}\right]$ defined by $v(R)=v R$ and the inverse mapping $\rho:\left[I, C_{0}\right] \rightarrow L R(X)$ defined by $\rho(V)=\rho V$ are dual isomorphisms.

\section{Theorem 3.4}

Let $X$ be a non-empty set. For $V \in\left[I, C_{0}\right]$ and $\left.p \in S((X \times X)-\Delta)\right)$, let $R_{p, V}=p(\rho V-\Delta) \cup \Delta$. Then $R_{p, V} \in$ $L R(X)$. Further, let $T_{p} V=v R_{p, v}$. Then $T_{p} V \in\left[I, C_{0}\right]$ and the mapping $T_{p}$ defined by $T_{p}(V)=T_{p} V$ for $V \in$ $\left[\mathrm{I}, \mathrm{C}_{0}\right]$ is an automorphism of $\left[\mathrm{I}, \mathrm{C}_{0}\right]$.

Proof:

From the Lemmas 3.1, $3.2 \&$ Remark 3.3, it follows that for $\mathrm{V}_{1}, \mathrm{~V}_{2} \in\left[\mathrm{I}, \mathrm{C}_{0}\right]$,

$$
\begin{aligned}
\mathrm{V}_{1} \leq \mathrm{V}_{2} & \Leftrightarrow \rho \mathrm{V}_{2} \subseteq \rho \mathrm{V}_{1} \\
& \Leftrightarrow \quad \mathrm{R}_{\mathrm{p}, \mathrm{V} 2} \subseteq \mathrm{R}_{\mathrm{p}, \mathrm{V} 1} \\
& \Leftrightarrow \quad \mathrm{T}_{\mathrm{p}} \mathrm{V}_{1} \leq \mathrm{T}_{\mathrm{p}} \mathrm{V}_{2} \\
& \Leftrightarrow \quad \mathrm{T}_{\mathrm{p}}\left(\mathrm{V}_{1}\right) \leq \mathrm{T}_{\mathrm{p}}\left(\mathrm{V}_{2}\right)
\end{aligned}
$$


Further, since the correspondences $\mathrm{V} \rightarrow \rho \mathrm{V}, \rho \mathrm{V} \rightarrow \rho \mathrm{V}-\Delta, \rho \mathrm{V}-\Delta \rightarrow \mathrm{p}(\rho \mathrm{V}-\Delta), \mathrm{p}(\rho \mathrm{V}-\Delta) \rightarrow \mathrm{p}(\rho \mathrm{V}-\Delta)$ $\cup \Delta\left(=R_{p, V}\right)$ and $R_{p, V} \rightarrow T_{p} V$ are bijections, it follows that $T_{p}: V \rightarrow T_{p} V$ is a bijection.

Hence $T_{p}$ is an automorphism of the lattice $\left[I, C_{0}\right]$.

Remark 3.5

Obviously the set of atoms of the lattice $\left[\mathrm{I}, \mathrm{C}_{0}\right]$ is precisely the set $\Omega=\left\{\mathrm{V}_{(\mathrm{a}, \mathrm{b})}: \mathrm{a}, \mathrm{b} \in \mathrm{X}\right.$ and $\left.\mathrm{a} \neq \mathrm{b}\right\}$.

Theorem 3.6

Let $\mathrm{X}$ be a non-empty finite set. Then the lattice $\left[\mathrm{I}, \mathrm{C}_{0}\right]$ coincides with the lattice $\mathrm{LC}(\mathrm{X})$ of all Cech closure operators on $X$ and hence the group of the lattice $\left[I, C_{0}\right]$ is precisely the set $\left\{T_{p}: p \in S((X \times X)-\Delta)\right\}$.

Proof:

Let $A$ be any automorphism of the lattice $\left[I, C_{0}\right]$. We want to show that $A=T_{p}$ for some $p \in S((X \times X)-\Delta)$. For $\mathrm{V}_{(\mathrm{a}, \mathrm{b})} \in \Omega$, let $\mathrm{A}\left(\mathrm{V}_{(\mathrm{a}, \mathrm{b})}\right)=\mathrm{V}_{(\mathrm{a}, \mathrm{b})^{\prime}}$ for some $(\mathrm{a}, \mathrm{b})^{\prime} \in(\mathrm{X} \times \mathrm{X})-\Delta$. Then $(\mathrm{a}, \mathrm{b})^{\prime}$ is unique. Define $\mathrm{p}(\mathrm{a}, \mathrm{b})=$ $(\mathrm{a}, \mathrm{b})^{\prime}$. Then $\mathrm{p} \in \mathrm{S}((\mathrm{X} \times \mathrm{X})-\Delta)$.

Now for $\mathrm{V}_{(\mathrm{a}, \mathrm{b})} \in \Omega$,

$$
\begin{aligned}
\mathrm{A}\left(\mathrm{V}_{(\mathrm{a}, \mathrm{b})}\right) & =\mathrm{V}_{(\mathrm{a}, \mathrm{b})^{\prime}} \\
& =v\left[\left(\rho \mathrm{V}_{\mathrm{p}(\mathrm{a}, \mathrm{b})}-\Delta\right) \cup \Delta\right] \\
& =v\left[\mathrm{p}\left(\rho \mathrm{V}_{(\mathrm{a}, \mathrm{b})}-\Delta\right) \cup \Delta\right] \\
& =\mathrm{T}_{\mathrm{p}} \mathrm{V}_{(\mathrm{a}, \mathrm{b})} \\
& =\mathrm{T}_{\mathrm{p}}\left(\mathrm{V}_{(\mathrm{a}, \mathrm{b})}\right)
\end{aligned}
$$

Hence $A=T_{p}$ on $\Omega$. Since $X$ is finite, the lattice $\left[I, C_{0}\right]=L C(X)$ is atomistic and hence it follows that $\mathrm{A}=\mathrm{T}_{\mathrm{p}}$ on $\left[\mathrm{I}, \mathrm{C}_{0}\right]$.

\section{Acknowledgements}

The author wishes to thank Dr. P.T. Ramachandran, Department of Mathematics, University of Calicut for his valuable guidance during the preparation of this paper.

\section{References}

[1] Baby Chacko, Some Lattice Theoretic Problems Related to Set Topology and Fuzzy Topology, Thesis for Ph.D. Degree, University of Calicut, Kerala., 2008

[2] Cech Edward , Topological Spaces, Rev. Ed.Wiley, New York, 1966.

[3] Garrett Birkhoff, Lattice Theory, Amer. Math. Soc. Colloq. Publ. Vol.25, Amer. Math.Soc. Providence, 1967.

[4] Juris Hartmanis, On the Lattice of Topologies, Cand. J . Math. 10 (1958), 547-553.

[5] Otto Frolich, Das Halbordnungssystem derTopologischen Raume auf einer Menge, Math. Ann. 156, (1964), 79-85.

[6] Ramachandran P. T. , Some Problems in Set Topology Relating Group of Homeomorphisms and Order, Thesis for Ph.D. Degree, Cochin University of Science and Technology, 1985.

[7] Stephen Willard, General Topology, Addison Wesley Publishing Co. 1970. 